Article

\title{
The Relationship between Serum 25-Hydroxyvitamin D Concentration, Cardiorespiratory Fitness, and Insulin Resistance in Japanese Men
}

\section{Xiaomin Sun ${ }^{1}$, Zhen-Bo Cao ${ }^{2,3, *}$, Kumpei Tanisawa ${ }^{1}$, Tomoko Ito ${ }^{1}$, Satomi Oshima ${ }^{3}$ and Mitsuru Higuchi ${ }^{3}$}

1 Graduate School of Sport Sciences, Waseda University, Tokorozawa, Saitama 359-1192, Japan; E-Mails: gzhtxiaomin@ruri.waseda.jp (X.S.); kunpei-tanisawa@fuji.waseda.jp (K.T.); maruto7691@moegi.waseda.jp (T.I.)

2 School of Kinesiology, Shanghai University of Sport, 399 Chang Hai Road, Shanghai 200438, China

3 Faculty of Sport Sciences, Waseda University, Tokorozawa, Saitama 359-1192, Japan; E-Mails: satomioshima@fuji.waseda.jp (S.O.); mhiguchi@waseda.jp (M.H.)

* Author to whom correspondence should be addressed; E-Mail: caozb_edu@yahoo.co.jp; Tel.: +86-21-5125-3257; Fax: +86-21-5125-3380.

Received: 26 November 2014 / Accepted: 15 December 2014 / Published: 29 December 2014

\begin{abstract}
Here, we aim to investigate the independent and combined associations of serum 25-hydroxyvitamin D (25(OH)D) and cardiorespiratory fitness (CRF) with glucose metabolism. Fasting blood samples of 107 men aged 40-79 years were analyzed for $25(\mathrm{OH}) \mathrm{D}$, glucose, insulin, glycated hemoglobin, and lipid profile. Homeostasis model assessment of insulin resistance index (HOMA-IR) was calculated from the fasting concentrations of glucose and insulin. Visceral fat area (VFA) was determined by magnetic resonance imaging and CRF by measuring maximal oxygen uptake. Median 25(OH)D concentration was $36.3 \mathrm{nmol} / \mathrm{L}$, while the prevalence of $25(\mathrm{OH}) \mathrm{D}$ deficiency was $74.8 \%$. Participants with high CRF had significantly lower HOMA-IR, glycated hemoglobin, and insulin values than participants with low CRF $(p<0.05)$. Higher $25(\mathrm{OH}) \mathrm{D}$ concentration was strongly correlated with lower HOMA-IR and insulin values independent of VFA $(p<0.01)$ but significantly affected by CRF. In the high CRF group, participants with higher $25(\mathrm{OH}) \mathrm{D}$ concentration had lower HOMA-IR values than participants with low 25(OH)D concentration $(p<0.05)$. Higher $25(\mathrm{OH}) \mathrm{D}$ and $\mathrm{CRF}$ are crucial for reducing insulin resistance regardless of abdominal fat. In addition, higher $25(\mathrm{OH}) \mathrm{D}$ concentration may strengthen the effect of CRF on reducing insulin resistance in middle-aged and elderly Japanese men with high CRF.
\end{abstract}


Keywords: vitamin D; cardiorespiratory fitness; insulin resistance; visceral fat

\section{Introduction}

Obesity and overweight are the most important risk factors for insulin resistance and type 2 diabetes mellitus (T2DM) [1,2]. In contrast to Caucasians with T2DM who always have high body mass indices (BMI), the Japanese population tends to develop T2DM with low BMI $[3,4]$. Therefore, besides obesity, other lifestyle factors, such as regular physical activity or ensuring sufficient micronutrient intake, may play an important role in the prevention of insulin resistance or T2DM in Japan.

Vitamin D is of vital importance for bone health and also appears to have extra-skeletal effects [5-8]. Recent evidence showed that high circulating 25-hydroxyvitamin D (25(OH)D) was associated with low prevalence of increased homeostasis model assessment of insulin resistance (HOMA-IR) and T2DM not only in individuals with impaired glucose tolerance or T2DM [9,10], but also in healthy individuals [11-14]; these associations were independent of BMI. In addition, several studies documented an association between high cardiorespiratory fitness (CRF) and low prevalence of insulin resistance and T2DM in adults, even after adjustment for BMI [15-22]. However, the possible interaction between circulating $25(\mathrm{OH}) \mathrm{D}$ and $\mathrm{CRF}$ with regard to insulin resistance has not been studied.

Therefore, the purpose of this study was to investigate the independent and combined associations of serum 25(OH)D and CRF levels with insulin resistance in middle-aged and elderly Japanese men.

\section{Materials and Methods}

\subsection{Subjects}

One hundred and seven Japanese men aged 40-79 years participated in this study. All procedures were conducted in Tokorozawa campus, Waseda University $\left(35^{\circ} \mathrm{N}\right.$ latitude). None of the participants had been diagnosed with cardiac disease, diabetes, or chronic renal failure. We excluded participants who were on lipid and glucose lowering medications, or medications that could affect the study variables (i.e., vitamin D supplements, vitamin D analogues, calcium, or any drugs that could affect bone and mineral metabolism, including bisphosphonates). We also recorded medication use including antihypertensive drugs that potentially affect glucose metabolism; 21 participants (19.6\%) were treated with antihypertensive drugs. Current/former smoking status was assessed by a questionnaire. Daily alcohol and vitamin D intake were assessed using a brief-type self-administered diet history questionnaire [23]. All participants provided written informed consent before enrollment in the study, which was approved by the Ethical Committee of Waseda University. The study was conducted in accordance with the Declaration of Helsinki. 


\subsection{Anthropometric Characteristics}

Body weight was measured by an electronic scale (Inner Scan BC-600, Tanita Inc., Tokyo, Japan), whereas height was measured by a stadiometer (YL-65, Yagami Inc., Nagoya, Japan). BMI was calculated from measurements of body weight and height. Visceral fat area (VFA) and subcutaneous fat area (SFA) were measured by magnetic resonance imaging (Signa 1.5T, General Electric Inc., Milwaukee, WI, USA). The imaging conditions included a T1-weighted spin-echo and axial-plane sequence with a slice thickness of $10 \mathrm{~mm}$, repetition time of $140 \mathrm{~ms}$, and echo time of $12.3 \mathrm{~ms}$ [22]. Cross-sectional images were scanned at the umbilical region. During the scan, the participants were asked to hold their breath for approximately $30 \mathrm{~s}$ after inhaling to reduce respiratory motion artifacts. Magnetic resonance images were transferred to a personal computer in the Digital Imaging and Communications in Medicine (DICOM) file format, and the cross-sectional VFA at the umbilical region was determined using image-analysis software (Slice-o-matic 4.3 for Windows, Tomovision, Montreal, QC, Canada). To minimize interobserver variation, all analyses were performed by the same investigator; the coefficient of variation was $0.4 \%$ for the cross-sectional areas of the umbilical region.

\subsection{Cardiorespiratory Fitness}

CRF was assessed by a maximal graded exercise test using a cycle ergometer (Ergomedic 828E; Monark, Varberg, Sweden) and quantified as maximal oxygen uptake $\left(\mathrm{VO}_{2} \mathrm{max}\right)$. The graded cycle exercise began at a workload of 45-90 W, which was increased by $15 \mathrm{~W} / \mathrm{min}$ until the subject could no longer maintain the required pedaling frequency of $60 \mathrm{rpm}$. Heart rate and ratings of perceived exertion were monitored each minute during exercise. During the incremental portion of the exercise test, expired gas was collected from the participants. $\mathrm{O}_{2}$ and $\mathrm{CO}_{2}$ concentrations were measured and averaged over $30 \mathrm{~s}$ intervals by an automated gas analyzer (Aeromonitor AE-300; Minato Medical Science, Tokyo, Japan). The maximum $\dot{\mathrm{VO}}_{2}$ recorded during the exercise test was considered the $\dot{\mathrm{VO}}_{2} \mathrm{max}(\mathrm{mL} / \mathrm{kg} / \mathrm{min})$, and the achievement of $\dot{\mathrm{VO}}_{2}$ max was accepted if at least 3 of the following 4 criteria were met: the $\dot{\mathrm{VO}}_{2}$ curve showed a plateau despite increasing the work rate, maximal heart rate was $95 \%$ of the age-predicted maximal heart rate (220-age (in years)), respiratory exchange ratio $>1.1$, and perceived exertion $\geq 18$. Participants were subsequently divided into the low and high CRF groups according to the median $\mathrm{VO}_{2}$ max value of each age group ( $\mathrm{mL} / \mathrm{kg} / \mathrm{min}$ ): 37.1 for $40-49$ years, 38.8 for 50-59 years, 31.3 for $60-69$ years, and 27.7 for $70-79$ years.

\subsection{Blood Sample Collection and Analysis}

Blood samples were collected between 08:30 and 11:00 AM by accredited nurses or doctors after a 12 -h overnight fast, and centrifuged at $3000 \times \mathrm{g}$ for $15 \mathrm{~min}$ at $4{ }^{\circ} \mathrm{C}$. Glucose, insulin, glycated hemoglobin $(\mathrm{HbA} 1 \mathrm{c})$, total cholesterol, high-density lipoprotein (HDL) cholesterol, low-density lipoprotein (LDL) cholesterol, and triglyceride concentrations were directly determined from fresh blood samples by BML Inc. (Tokyo, Japan). The HOMA-IR value was used as an index of insulin resistance; it was calculated from the fasting concentrations of plasma glucose and serum insulin as follows:

HOMA-IR $=[$ fasting glucose $(\mathrm{mg} / \mathrm{dL})] \times[$ fasting insulin $(\mu \mathrm{U} / \mathrm{mL})] / 405$ 
Serum 25(OH)D concentration was measured in duplicate using commercially available enzyme-linked immunosorbent assay kits $(25(\mathrm{OH}) \mathrm{D}$ : Immundiagnostik AG, Bensheim, Germany) according to the manufacturer's instructions. The intra- and interassay coefficients of variation were $8.9 \%$ and $10.6 \%$ for $25(\mathrm{OH}) \mathrm{D}$. We divided participants into low and high $25(\mathrm{OH}) \mathrm{D}$ groups according to the median values of $25(\mathrm{OH}) \mathrm{D}$ concentration $(36.3 \mathrm{nmol} / \mathrm{L})$.

\subsection{Statistical Analysis}

All statistical analyses were performed using SPSS version 22.0 (SPSS, Inc., Chicago, IL, USA). Kolmogorov-Smirnov test was performed to assess the normality of data distribution, and several variables were log-transformed or square root transformed to obtain a normal distribution prior to analysis. Student's $t$-test (for normal distributed variables), Mann-Whitney $U$-test (for non-normally distributed variables), or chi-square test (for categorical variables) was used to evaluate the significance of differences between the low and high CRF groups. Partial correlation analysis adjusted for age, season or VFA was performed to determine the associations between $25(\mathrm{OH}) \mathrm{D}$ concentration and subject characteristics. The influence of CRF levels and 25(OH)D concentration on blood parameters was evaluated by two-way analysis of covariance (ANCOVA) adjusted for the appropriate covariates. A post hoc test with Bonferroni correction was used to identify significant differences if a significant main effect or interaction was identified. Fisher's exact test was used for comparing proportions of participants with high risk of insulin resistance (HOMA-IR $\geq 1.6$ ) between combination groups. For this analysis, we created 4 subgroups for combinations of $25(\mathrm{OH}) \mathrm{D}$ and $\mathrm{CRF}$ on the basis of dichotomizing groups with low and high levels of each variable (High CRF and 25(OH)D subgroup, HH; High CRF and Low 25(OH)D subgroup, HL; Low CRF and High 25(OH)D subgroup, LH; Low CRF and 25(OH)D subgroup, LL). All measurements and calculated values are presented as mean (SD) (for normally distributed variables) or median (interquartile ratio; IQR) (for non-normally distributed variables) unless otherwise indicated. The level of significance was set at $p<0.05$.

\section{Results}

The characteristics of study participants are shown in Table 1 . The median 25(OH)D concentration was 36.3 (IQR: $26.4-50.2) \mathrm{nmol} / \mathrm{L}, 74.8 \%$ of participants were $25(\mathrm{OH}) \mathrm{D}$ deficient $(<50 \mathrm{nmol} / \mathrm{L})$, and $13.1 \%$ of participants had insufficient $25(\mathrm{OH}) \mathrm{D}(50-75 \mathrm{nmol} / \mathrm{L})$. The median age of participants was 67.0 years for the low CRF group and 65.0 years for the high CRF group. The low CRF group had lower CRF, HDL cholesterol, 25(OH)D concentration, and vitamin D intake values and higher VFA, HbA1c, insulin and HOMA-IR values $(p<0.05)$ than the high CRF group. In addition, the low CRF group had slightly higher triglyceride levels than the high CRF group but was not statistically significant $(p=0.052)$. 
Table 1. Subject characteristics.

\begin{tabular}{|c|c|c|c|c|c|c|c|}
\hline \multirow{2}{*}{$\begin{array}{c}\text { Variable } \\
\text { Age (years) }\end{array}$} & \multicolumn{2}{|c|}{$\begin{array}{c}\text { All } \\
(n=107)\end{array}$} & \multicolumn{2}{|c|}{$\begin{array}{c}\text { Low CRF } \\
(n=53)\end{array}$} & \multicolumn{2}{|c|}{$\begin{array}{c}\text { High CRF } \\
(n=54)\end{array}$} & \multirow{2}{*}{$\frac{p}{0.300}$} \\
\hline & 65.0 & $(61.0-70.0)$ & 67.0 & $(61.5-71.0)$ & 65.0 & $(60.8-70.0)$ & \\
\hline Height (cm) & 168.8 & $(6.5)$ & 167.6 & (5.7) & 170.0 & (7.1) & 0.057 \\
\hline Weight (kg) & 66.8 & $(8.7)$ & 66.4 & $(8.8)$ & 67.1 & $(8.7)$ & 0.684 \\
\hline BMI $\left(\mathrm{kg} / \mathrm{m}^{2}\right)$ & 23.4 & (2.3) & 23.6 & (2.4) & 23.1 & $(2.1)$ & 0.328 \\
\hline $\operatorname{VFA}\left(\mathrm{cm}^{2}\right)$ & 109.5 & $(46.9)$ & 119.4 & $(48.2)$ & 99.8 & (43.4) & 0.029 \\
\hline $\operatorname{SFA}\left(\mathrm{cm}^{2}\right)$ & 82.5 & $(58.5-120.9)$ & 77.8 & $(58.8-127.3)$ & 91.1 & $(56.1-113.7)$ & 0.528 \\
\hline $\mathrm{CRF}(\mathrm{mL} / \mathrm{kg} / \mathrm{min})$ & 32.3 & $(27.4-36.2)$ & 27.4 & $(22.8-29.8)$ & 35.4 & $(32.5-39.1)$ & $<0.001$ \\
\hline Glucose (mg/dL) & 98.2 & (9.6) & 99.0 & (11.3) & 97.4 & (7.6) & 0.396 \\
\hline HbA1c (\%) & 5.0 & $(0.3)$ & 5.1 & $(0.3)$ & 4.9 & $(0.2)$ & 0.032 \\
\hline Insulin $(\mu \mathrm{U} / \mathrm{mL})$ & 5.0 & $(3.5-6.5)$ & 5.8 & $(4.0-8.1)$ & 4.3 & $(3.0-5.9)$ & 0.002 \\
\hline HOMA-IR & 1.14 & $(0.86-1.66)$ & 1.37 & $(0.94-1.94)$ & 1.04 & $(0.72-1.37)$ & 0.002 \\
\hline Total cholesterol (mg/dL) & 213.9 & $(32.9)$ & 213.5 & $(34.0)$ & 214.2 & $(32.1)$ & 0.907 \\
\hline HDL cholesterol (mg/dL) & 61.0 & $(52.0-68.0)$ & 59.0 & $(50.0-65.0)$ & 64.0 & $(54.0-70.0)$ & 0.028 \\
\hline LDL cholesterol (mg/dL) & 122.5 & $(30.3)$ & 124.4 & $(32.5)$ & 120.6 & $(28.2)$ & 0.529 \\
\hline Triglycerides (mg/dL) & 91.0 & $(65.0-119.0)$ & 97.0 & $(67.5-139.5)$ & 83.0 & $(62.0-104.3)$ & 0.052 \\
\hline $25(\mathrm{OH}) \mathrm{D}(\mathrm{nmol} / \mathrm{L})$ & 36.3 & $(26.4-50.2)$ & 33.2 & $(22.0-38.8)$ & 45.3 & $(29.6-66.8)$ & $<0.001$ \\
\hline Vitamin D intake ( $\mu \mathrm{g} /$ day) & 12.6 & $(8.9-18.8)$ & 10.8 & $(8.5-16.9)$ & 14.2 & $(9.3-24.5)$ & 0.034 \\
\hline Alcohol consumption (g/day) & 21.5 & $(8.8-42.2)$ & 15.7 & $(0.8-36.1)$ & 25.4 & $(9.8-49.3)$ & 0.106 \\
\hline Smoking status (\%) & & 51.4 & & 60.4 & & 42.6 & 0.066 \\
\hline Medication use (\%) & & 29.0 & & 28.3 & & 29.6 & 0.880 \\
\hline
\end{tabular}

Data are mean (SD) or median (IQR) values. BMI: body mass index; VFA: visceral fat area; SFA: subcutaneous fat area; CRF: cardiorespiratory fitness quantified as $\mathrm{VO}_{2}$ max; HbAlc: glycated hemoglobin; HOMA-IR: homeostasis model assessment of insulin resistance; HDL: high-density lipoprotein; LDL: low-density lipoprotein; 25(OH)D: 25-hydroxyvitamin D.

Table 2 shows the correlation between 25(OH)D concentration and other variables. Results showed that $25(\mathrm{OH}) \mathrm{D}$ concentration was positively correlated with HDL cholesterol, vitamin D intake and CRF, and negatively correlated with insulin, HOMA-IR, triglycerides, and VFA $(p<0.05)$, after adjusting for age and season. Moreover, the relationships persisted after further adjustment for VFA.

To evaluate interaction effects between 25(OH)D and CRF on the blood parameters, two-way ANCOVA was performed after adjustment for potential confounders, including VFA (Table 3 and Figure 1). Significant interactions between 25(OH)D and CRF on insulin and HOMA-IR values were found $(p<0.05)$. No significant interactions were observed on glucose, HbA1c, triglycerides, and LDL cholesterol levels. We found significant main effects on insulin and HOMA-IR values for CRF ( $p=0.034$ and 0.033 , respectively), but not for $25(\mathrm{OH}) \mathrm{D}$. In the high CRF group (more than the median values of each age group), participants with high $25(\mathrm{OH}) \mathrm{D}$ concentration $(\geq 36.3 \mathrm{nmol} / \mathrm{L})$ had lower insulin $(p=0.038)$ and HOMA-IR $(p=0.043)$ values than participants with low 25(OH)D concentration $(<36.3 \mathrm{nmol} / \mathrm{L})$. In the high $25(\mathrm{OH}) \mathrm{D}$ group, participants with high CRF had lower insulin $(p=0.002)$ and HOMA-IR $(p=0.001)$ values than participants with low CRF. In addition, participants simultaneously in the high CRF and $25(\mathrm{OH}) \mathrm{D}$ subgroup $(\mathrm{HH})$ had the lowest prevalence of insulin 
resistance (HOMA-IR $\geq 1.6)$ among all combination subgroups ( $8.6 \%$ for $\mathrm{HH}, 31.6 \%$ for $\mathrm{HL}, 47.4 \%$ for LH, 32.4\% for LL, $p<0.05)$.

Table 2. Correlations of serum $25(\mathrm{OH}) \mathrm{D}$ with subject characteristics in middle-aged and elderly adults.

\begin{tabular}{ccccc}
\hline & \multicolumn{2}{c}{$\begin{array}{c}\mathbf{2 5}(\mathbf{O H}) \mathbf{D} \text { (Age- and } \\
\text { Season-Adjusted) }\end{array}$} & $\begin{array}{r}\mathbf{2 5}(\mathbf{O H}) \mathbf{D} \text { (Age- } \text { Season- and } \\
\text { VFA-Adjusted) }\end{array}$ \\
\cline { 2 - 5 } & $\boldsymbol{r}$ & $\boldsymbol{p}$ & $\boldsymbol{r}$ & $\boldsymbol{p}$ \\
\hline BMI $\left(\mathrm{kg} / \mathrm{m}^{2}\right)$ & -0.033 & 0.742 & 0.127 & 0.198 \\
VFA $\left(\mathrm{cm}^{2}\right)$ & -0.199 & 0.042 & & \\
SFA $\left(\mathrm{cm}^{2}\right)$ & 0.022 & 0.822 & 0.152 & 0.124 \\
CRF $(\mathrm{mL} / \mathrm{kg} / \mathrm{min})$ & 0.370 & $<0.001$ & 0.340 & $<0.001$ \\
Glucose $(\mathrm{mg} / \mathrm{dL})$ & -0.009 & 0.929 & 0.000 & 0.999 \\
HbA1c $(\%)$ & -0.134 & 0.171 & -0.114 & 0.249 \\
Insulin $(\mu \mathrm{H} / \mathrm{mL})$ & -0.348 & $<0.001$ & -0.301 & 0.002 \\
HOMA-IR & -0.331 & 0.001 & -0.283 & 0.004 \\
Total cholesterol $(\mathrm{mg} / \mathrm{dL})$ & -0.015 & 0.879 & -0.027 & 0.782 \\
HDL cholesterol $(\mathrm{mg} / \mathrm{dL})$ & 0.247 & 0.011 & 0.198 & 0.044 \\
LDL cholesterol $(\mathrm{mg} / \mathrm{dL})$ & -0.087 & 0.375 & -0.081 & 0.416 \\
Triglycerides $(\mathrm{mg} / \mathrm{dL})$ & -0.304 & 0.002 & -0.251 & 0.010 \\
Vitamin D intake $(\mu \mathrm{gg} / \mathrm{day})$ & 0.299 & 0.002 & 0.280 & 0.004 \\
\hline
\end{tabular}

BMI: body mass index; VFA: visceral fat area; SFA: subcutaneous fat area; CRF: cardiorespiratory fitness quantified as VO2max; HbA1c: glycated hemoglobin; HOMA-IR: homeostasis model assessment of insulin resistance; HDL: high-density lipoprotein; LDL: low-density lipoprotein; 25(OH)D: 25-hydroxyvitamin D. Partial Pearson's correlation coefficients were calculated. SFA, insulin, HOMA-IR, HDL cholesterol, triglycerides, and vitamin D intake were log-transformed; 25(OH)D was square root transformed for analysis.

Table 3. Joint association of 25(OH)D and CRF with parameters related to insulin resistance.

\begin{tabular}{|c|c|c|c|c|c|c|c|}
\hline \multirow{3}{*}{ Blood Variable } & \multicolumn{2}{|c|}{ Low CRF } & \multicolumn{2}{|c|}{ High CRF } & \multirow{3}{*}{$\begin{array}{c}\text { CRF } \\
p\end{array}$} & \multirow{3}{*}{$\begin{array}{c}\text { 25(OH)D } \\
p\end{array}$} & \multirow{3}{*}{$\begin{array}{c}\text { Interaction } \\
p\end{array}$} \\
\hline & Low 25(OH)D & High 25(OH)D & Low 25(OH)D & High 25(OH)D & & & \\
\hline & $(n=34)$ & $(n=19)$ & $(n=19)$ & $(n=35)$ & & & \\
\hline Glucose $(\mathrm{mg} / \mathrm{dL})$ & $98.1 \pm 1.6$ & $100.6 \pm 2.2$ & $98.2 \pm 2.3$ & $97.1 \pm 1.7$ & 0.409 & 0.743 & 0.343 \\
\hline HbA1c (\%) & $5.06 \pm 0.05$ & $5.06 \pm 0.06$ & $5.02 \pm 0.07$ & $4.92 \pm 0.05$ & 0.115 & 0.433 & 0.340 \\
\hline Insulin $(\mu \mathrm{U} / \mathrm{mL})$ & $5.2 \pm 1.08$ & $6.03 \pm 1.1$ & $5.3 \pm 1.11$ & $4.02 \pm 1.08 *, \uparrow$ & 0.034 & 0.494 & 0.016 \\
\hline HOMA-IR & $1.25 \pm 1.08$ & $1.49 \pm 1.11$ & $1.28 \pm 1.11$ & $0.96 \pm 1.08 *, \dagger$ & 0.033 & 0.565 & 0.014 \\
\hline Triglycerides (mg/dL) & $103.5 \pm 1.1$ & $84 \pm 1.1$ & $88.9 \pm 1.1$ & $84.5 \pm 1.1$ & 0.458 & 0.200 & 0.403 \\
\hline LDL cholesterol (mg/dL) & $126.9 \pm 5.4$ & $120.7 \pm 7.2$ & $118 \pm 7.5$ & $121.5 \pm 5.5$ & 0.541 & 0.842 & 0.442 \\
\hline HDL cholesterol (mg/dL) & $60.3 \pm 1$ & $56.9 \pm 1.1$ & $57.8 \pm 1.1$ & $64.6 \pm 1$ & 0.368 & 0.582 & 0.058 \\
\hline
\end{tabular}

CRF: cardiorespiratory fitness quantified as $\mathrm{VO}_{2}$ max; $\mathrm{HbAlc}$ : glycated hemoglobin; HOMA-IR: homeostasis model assessment of insulin resistance; HDL: high-density lipoprotein; LDL: low-density lipoprotein; 25(OH)D: 25-hydroxyvitamin D. Data are presented as the adjusted mean \pm SE. Insulin, HOMA-IR, triglycerides and HDL cholesterol were log transformed (data are shown as adjusted geometric mean $\pm \mathrm{SE}$ ). Data were analyzed using two-way analysis of covariance (ANCOVA) adjusted for age, season, VFA, smoking status, medication use, vitamin D intake, and alcohol consumption. * $p<0.05$ vs. low CRF within the same $25(\mathrm{OH}) \mathrm{D}$ group. ${ }^{\dagger} p<0.05 v s$. low 25(OH)D within the same CRF group. In the present study, 25(OH)D groups were divided according to the median serum 25(OH)D concentration (36.3 nmol/L) and CRF groups were divided according to the median $\dot{\mathrm{VO}}_{2}$ max value of each age group (mL/kg/min): 37.1 for $40-49$ years, 38.8 for $50-59$ years, 31.3 for $60-69$ years, and 27.7 for $70-79$ years. 


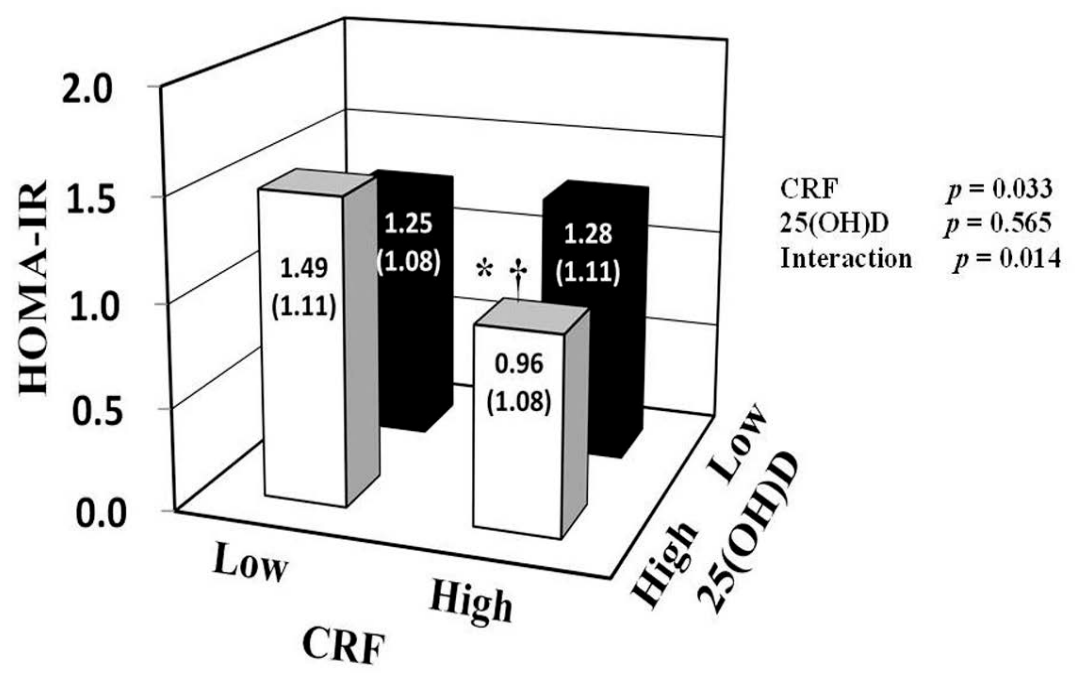

Figure 1. Associations of $25(\mathrm{OH}) \mathrm{D}$ and CRF with HOMA-IR. HOMA-IR was log transformed for two-way analysis of covariance with adjustment for age, season, visceral fat area, smoking status, medication use, vitamin D intake, and alcohol consumption. Data are shown as adjusted geometric mean \pm SE. ${ }^{*} p<0.05 v s$. low CRF within the same $25(\mathrm{OH}) \mathrm{D}$ group. ${ }^{\dagger} p<0.05$ vs. low $25(\mathrm{OH}) \mathrm{D}$ within the same CRF group. In the present study, $25(\mathrm{OH}) \mathrm{D}$ groups were divided according to the median serum $25(\mathrm{OH}) \mathrm{D}$ concentration (36.3 nmol/L), and CRF groups were divided according to the median $\mathrm{VO}_{2}$ max value of each age group (mL/kg/min): 37.1 for 40-49 years, 38.8 for 50-59 years, 31.3 for 60-69 years, and 27.7 for 70-79 years. CRF: cardiorespiratory fitness; HOMA-IR: homeostasis model assessment of insulin resistance; 25(OH)D: 25-hydroxyvitamin D.

\section{Discussion}

To our knowledge, this study was the first to examine the combined associations of $25(\mathrm{OH}) \mathrm{D}$ and CRF with glucose metabolism in middle-aged and elderly Japanese men. Our results indicate that higher 25(OH)D concentration and CRF levels were associated with low risk of insulin resistance, independent of VFA levels. Furthermore, among participants with high CRF levels, higher serum 25(OH)D concentration largely strengthened the effect of CRF on reducing insulin resistance.

Several studies have shown that high levels of CRF are associated with low insulin resistance or incidence of T2DM in healthy adults including Japanese [19-22,24]. Usui et al. [22] revealed that fasting insulin concentrations and HOMA-IR were significantly lower among the high CRF group than the low CRF group in healthy Japanese women and men. Moreover, data from the Tokyo Gas Company in Japan showed that high CRF groups have significantly lower risk of developing T2DM compared with other fitness groups, even after controlling for BMI in healthy Japanese men [21,24]. Although previous studies have considered obesity levels using BMI, recent studies have revealed that reductions in the VFA may occur in the absence of changes in BMI [25]. The present study clearly showed that participants with higher CRF had significantly lower HOMA-IR and insulin values, independent of VFA levels, which is a more robust measure of obesity than the simple anthropometric measurement BMI. It indicates that high levels of CRF are critical for attenuating insulin resistance in middle-aged and elderly Japanese men, regardless of visceral obesity. 
It has been reported that higher $25(\mathrm{OH}) \mathrm{D}$ concentration is strongly associated with low insulin and insulin resistance in adults $[13,14]$. However, those previous studies have been mostly limited to non-Asian populations. Furthermore, in place of directly measured CRF, self-reported physical activity determined by simple questionnaires was used as a controlled factor to determine the relationship between 25(OH)D concentration and HOMA-IR in those previous studies [7]. Although self-reported physical activity is related to CRF, prior evidence has demonstrated that CRF is much more strongly related with various health outcomes than self-reported physical activity, which often inevitably produces greater misclassification than the directly measured CRF [26-28]. In addition, the combined associations of serum $25(\mathrm{OH}) \mathrm{D}$ and $\mathrm{CRF}$ with glucose metabolism has not been examined. Consistent with the previous study $[13,21,22]$, the present study revealed that higher $25(\mathrm{OH}) \mathrm{D}$ concentration and CRF levels were related to lower levels of fasting insulin and insulin resistance, independent of obesity status (VFA levels), in middle-aged and elderly Japanese men. Additionally, the present study is the first to demonstrate an interaction effect of serum 25(OH)D and CRF on glucose metabolism in Japanese middle-aged and elderly men. We found the relationship between $25(\mathrm{OH}) \mathrm{D}$ concentration and HOMA-IR was significantly affected by CRF levels. This was not surprising since $25(\mathrm{OH}) \mathrm{D}$ was more closely related with CRF than VFA levels ( $r=0.370$ vs. $r=0.199)$. The positive association between $25(\mathrm{OH}) \mathrm{D}$ and CRF could be explained partly by the variations in daily physical activity, sunlight exposure time, and cardiac morphology [29,30]. Moreover, we found that the values of HOMA-IR and the number of participants with high risk of insulin resistance were lower in the combined higher $25(\mathrm{OH}) \mathrm{D}$ and $\mathrm{CRF}$ group than in the other groups $(p<0.05$ ). These observations suggest that the combination of higher CRF and $25(\mathrm{OH}) \mathrm{D}$ levels are probably more effective to reduce the risk of insulin resistance than either alone.

Although the clear mechanism is not well understood, several explanations can be given for this finding. Human and animal studies have reported that both higher levels of $25(\mathrm{OH}) \mathrm{D}$ and regular physical activity increased insulin secretion and enhanced insulin sensitivity in pancreatic and peripheral tissues [12,18,31]; thus, a combined effect of higher levels of 25(OH)D and CRF on glycemic control may be stronger than either alone. Additionally, it has been demonstrated that altered cholesterol metabolism (for example, low HDL cholesterol or high total cholesterol levels) may contribute to prevalence of insulin resistance [32], and regular physical activity and higher 25(OH)D concentration may improve lipid metabolism, resulting in low risk of insulin resistance [7,18,33]. Consistent with previous studies, the present study found that $25(\mathrm{OH}) \mathrm{D}$ and CRF are positively related to HDL cholesterol ( $p<0.05$ and $p<0.01$, respectively), independent of age, season and VFA levels. Additionally, the two-way ANCOVA showed that participants simultaneously in the higher CRF and 25(OH)D group had higher HDL cholesterol levels than other groups, though not statistically significant $(p=0.06)$. Thus, it seems reasonable to infer that the combined effect of higher levels of $25(\mathrm{OH}) \mathrm{D}$ and $\mathrm{CRF}$ on glucose control is more effective than either alone, at least in our study population.

The present study has several limitations. First, because this was a cross-sectional study, it is difficult to make causal inferences between exposures and outcomes. A prospective study would provide more accurate associations of $25(\mathrm{OH}) \mathrm{D}$ concentration and CRF levels with insulin resistance. Second, the present study evaluated insulin resistance on the basis of HOMA-IR, which uses fasting values for estimation and mainly reflects insulin resistance in the liver [34]. Although HOMA-IR has been shown to have a good relationship with the hyperinsulinemic euglycemic clamp technique [35], a more accurate method for assessing insulin resistance, such as the oral glucose tolerance test or the glycemic insulin 
clamp test, should be used in future studies. Third, despite individuals had a higher daily vitamin D intake $(12.6 \mu \mathrm{g} /$ day $)$ than Dietary Reference Intake (5.5 $\mu \mathrm{g}$ /day) for Japanese in the present study, the prevalence of $25(\mathrm{OH}) \mathrm{D}$ deficiency $(74.8 \%)$ is still high; therefore, whether the findings can be extrapolated to reflect serum $25(\mathrm{OH}) \mathrm{D}$ sufficiency needs to be investigated. Finally, because we only examined men in the present study, our results should be interpreted with caution and confirmed in further cohorts of women. Despite these limitations, our study was the first to evaluate the combined associations of serum 25(OH)D concentration and CRF levels with insulin resistance, controlling for potential confounding factors including a precise obesity indicator in middle-aged and elderly Japanese men.

\section{Conclusions}

In conclusion, the present study revealed that higher $25(\mathrm{OH}) \mathrm{D}$ concentration and CRF levels were negatively correlated with levels of fasting insulin and insulin resistance in middle-aged and elderly Japanese men. These associations were independent of abdominal fat, while association between serum 25(OH)D and insulin resistance was largely influenced by CRF levels. Among middle-aged and elderly Japanese men with high levels of CRF, higher 25(OH)D concentration largely enhanced the effect of $\mathrm{CRF}$ on reducing insulin resistance. These results suggest that the combination of increasing both CRF and circulating $25(\mathrm{OH}) \mathrm{D}$ may be more effective for improving glycemic control than either alone.

\section{Acknowledgments and Funding}

We would like to thank the subjects who participated in this study. Additionally, we would like to thank our laboratory members (Hiroshi Kawano, Yuko Gando, Ryoko Kawakami, Takafumi Ando, and Taishi Susa) who provided support for data collection. This study was supported in part by a Grant-in-Aid for the Global COE (Sport Sciences for the Promotion of Active Life to Waseda University) from the Ministry of Education, Culture, Sports, Science, and Technology of Japan (to Mitsuru Higuchi), Grants-in-Aid for Scientific Research (C) from the Ministry of Education, Culture, Sports, Science and Technology of Japan (to Zhen-Bo Cao, The Program for Professor of Special Appointment (Eastern Scholar) at Shanghai Institutions of Higher Learning (No. TP2014057), and Shanghai Key Lab of Human Performance (Shanghai University of sport) (No. 11DZ2261100). Xiaomin Sun (No. 14-349) was the recipient of a scholarship from the Ostuka Toshimi Scholarship Foundation.

\section{Author Contributions}

Xiaomin Sun and Zhen-Bo Cao designed the experiments. Xiaomin Sun performed statistical analyses and drafted the manuscript. Kumpei Tanisawa, Satomi Oshima, Tomoko Ito and Mitsuru Higuchi collected the research data. Zhen-Bo Cao and Mitsuru Higuchi critically reviewed and edited the manuscript. Zhen-Bo Cao conceived and supervised the entire study. All authors read and approved the final manuscript.

\section{Conflicts of interest}

The authors have no conflict of interest to declare. 


\section{References}

1. Caballero, B. The global epidemic of obesity: An overview. Epidemiol. Rev. 2007, 29, 1-5.

2. Pisunyer, F.X. Health implications of obesity. Am. J. Clin. Nutr. 1991, 53, S1595-S1603.

3. Yoon, K.H.; Lee, J.H.; Kim, J.W.; Cho, J.H.; Choi, Y.H.; Ko, S.H.; Zimmet, P.; Son, H.Y. Epidemic obesity and type 2 diabetes in Asia. Lancet 2006, 368, 1681-1688.

4. Sone, H.; Ito, H.; Ohashi, Y.; Akanuma, Y.; Yamada, N. Obesity and type 2 diabetes in Japanese patients. Lancet 2003, 361, 85.

5. Sun, X.; Cao, Z.B.; Zhang, Y.; Ishimi, Y.; Tabata, I.; Higuchi, M. Association between serum 25-hydroxyvitamin D and inflammatory cytokines in healthy adults. Nutrients 2014, 6, 221-230.

6. Parker, J.; Hashmi, O.; Dutton, D.; Mavrodaris, A.; Stranges, S.; Kandala, N.B.; Clarke, A.; Franco, O.H. Levels of vitamin D and cardiometabolic disorders: Systematic review and meta-analysis. Maturitas 2010, 65, 225-236.

7. Girgis, C.M.; Clifton-Bligh, R.J.; Turner, N.; Lau, S.L.; Gunton, J.E. Effects of vitamin D in skeletal muscle: Falls, strength, athletic performance and insulin sensitivity. Clin. Endocrinol. 2014, 80, 169-181.

8. Wacker, M.; Holick, M.F. Vitamin D-effects on skeletal and extraskeletal health and the need for supplementation. Nutrients 2013, 5, 111-148.

9. Scragg, R.; Holdaway, I.; Singh, V.; Metcalf, P.; Baker, J.; Dryson, E. Serum 25-hydroxyvitamin $\mathrm{D}_{3}$ levels decreased in impaired glucose tolerance and diabetes mellitus. Diabetes Res. Clin. Pract. 1995, 27, 181-188.

10. Cavalier, E.; Delanaye, P.; Souberbielle, J.C.; Radermecker, R.P. Vitamin D and type 2 diabetes mellitus: Where do we stand? Diabetes Metab. 2011, 37, 265-272.

11. Knekt, P.; Laaksonen, M.; Mattila, C.; Harkanen, T.; Marniemi, J.; Heliovaara, M.; Rissanen, H.; Montonen, J.; Reunanen, A. Serum vitamin D and subsequent occurrence of type 2 diabetes. Epidemiology 2008, 19, 666-671.

12. Gagnon, C.; Lu, Z.X.; Magliano, D.J.; Dunstan, D.W.; Shaw, J.E.; Zimmet, P.Z.; Sikaris, K.; Grantham, N.; Ebeling, P.R.; Daly, R.M. Serum 25-hydroxyvitamin D, calcium intake, and risk of type 2 diabetes after 5 years: Results from a national, population-based prospective study (the Australian diabetes, obesity and lifestyle study). Diabetes Care 2011, 34, 1133-1138.

13. Forouhi, N.G.; Luan, J.; Cooper, A.; Boucher, B.J.; Wareham, N.J. Baseline serum 25-hydroxy vitamin $\mathrm{D}$ is predictive of future glycemic status and insulin resistance: The medical research council ely prospective study 1990-2000. Diabetes 2008, 57, 2619-2625.

14. Liu, E.; Meigs, J.B.; Pittas, A.G.; McKeown, N.M.; Economos, C.D.; Booth, S.L.; Jacques, P.F. Plasma 25-hydroxyvitamin D is associated with markers of the insulin resistant phenotype in nondiabetic adults. J. Nutr. 2009, 139, 329-334.

15. Lee, D.C.; Sui, X.; Church, T.S.; Lee, I.M.; Blair, S.N. Associations of cardiorespiratory fitness and obesity with risks of impaired fasting glucose and type 2 diabetes in men. Diabetes Care 2009, 32, 257-262.

16. Yokoyama, H.; Emoto, M.; Araki, T.; Fujiwara, S.; Motoyama, K.; Morioka, T.; Koyama, H.; Shoji, T.; Okuno, Y.; Nishizawa, Y. Effect of aerobic exercise on plasma adiponectin levels and insulin resistance in type 2 diabetes. Diabetes Care 2004, 27, 1756-1758. 
17. Tanisawa, K.; Ito, T.; Sun, X.; Ise, R.; Oshima, S.; Cao, Z.B.; Sakamoto, S.; Tanaka, M.; Higuchi, M. High cardiorespiratory fitness can reduce glycated hemoglobin levels regardless of polygenic risk for type 2 diabetes mellitus in non-diabetic Japanese men. Physiol. Genomics 2014, 46, 497-504.

18. Borghouts, L.B.; Keizer, H.A. Exercise and insulin sensitivity: A review. Int. J. Sports Med. 2000, $21,1-12$.

19. Kawakami, R.; Sawada, S.S.; Matsushita, M.; Okamoto, T.; Tsukamoto, K.; Higuchi, M.; Miyachi, M. Reference values for cardiorespiratory fitness and incidence of type 2 diabetes. J. Epidemiol. 2014, 24, 25-30.

20. Sieverdes, J.C.; Sui, X.; Lee, D.C.; Church, T.S.; McClain, A.; Hand, G.A.; Blair, S.N. Physical activity, cardiorespiratory fitness and the incidence of type 2 diabetes in a prospective study of men. Br. J. Sports Med. 2010, 44, 238-244.

21. Sawada, S.S.; Lee, I.M.; Naito, H.; Noguchi, J.; Tsukamoto, K.; Muto, T.; Higaki, Y.; Tanaka, H.; Blair, S.N. Long-term trends in cardiorespiratory fitness and the incidence of type 2 diabetes. Diabetes Care 2010, 33, 1353-1357.

22. Usui, C.; Asaka, M.; Kawano, H.; Aoyama, T.; Ishijima, T.; Sakamoto, S.; Higuchi, M. Visceral fat is a strong predictor of insulin resistance regardless of cardiorespiratory fitness in non-diabetic people. J. Nutr. Sci. Vitaminol. 2010, 56, 109-116.

23. Murakami, K.; Mizoue, T.; Sasaki, S.; Ohta, M.; Sato, M.; Matsushita, Y.; Mishima, N. Dietary intake of folate, other B vitamins, and omega-3 polyunsaturated fatty acids in relation to depressive symptoms in Japanese adults. Nutrition 2008, 24, 140-147.

24. Sawada, S.S.; Lee, I.M.; Muto, T.; Matuszaki, K.; Blair, S.N. Cardiorespiratory fitness and the incidence of type 2 diabetes: Prospective study of Japanese men. Diabetes Care 2003, 26, 2918-2922.

25. Kay, S.J.; Fiatarone Singh, M.A. The influence of physical activity on abdominal fat: A systematic review of the literature. Obes. Rev. 2006, 7, 183-200.

26. Blair, S.N.; Cheng, Y.; Holder, J.S. Is physical activity or physical fitness more important in defining health benefits? Med. Sci. Sports Exerc. 2001, 33, s379-399.

27. Kampert, J.B.; Blair, S.N.; Barlow, C.E.; Kohl, H.W. III. Physical activity, physical fitness, and all-cause and cancer mortality: A prospective study of men and women. Ann. Epidemiol. 1996, 6, $452-457$.

28. Lee, D.C.; Sui, X.; Ortega, F.B.; Kim, Y.S.; Church, T.S.; Winett, R.A.; Ekelund, U.; Katzmarzyk, P.T.; Blair, S.N. Comparisons of leisure-time physical activity and cardiorespiratory fitness as predictors of all-cause mortality in men and women. Br. J. Sports Med. 2011, 45, 504-510.

29. Nibbelink, K.A.; Tishkoff, D.X.; Hershey, S.D.; Rahman, A.; Simpson, R.U. 1,25(OH)2D vitamin D3 actions on cell proliferation, size, gene expression, and receptor localization, in the HL-1 cardiac myocyte. J. Steroid Biochem. Mol. Biol. 2007, 103, 533-537.

30. Scragg, R.; Camargo, C.A. Frequency of leisure-time physical activity and serum 25-hydroxyvitamin D levels in the US population: Results from the third national health and nutrition examination survey. Am. J. Epidemiol. 2008, 168, 577-586.

31. Chiu, K.C.; Chu, A.; Go, V.L.; Saad, M.F. Hypovitaminosis D is associated with insulin resistance and $\beta$ cell dysfunction. Am. J. Clin. Nutr. 2004, 79, 820-825. 
32. Zhang, L.; Qiao, Q.; Tuomilehto, J.; Hammar, N.; Alberti, K.G.M.M.; Eliasson, M.; Heine, R.J.; Stehouwer, C.D.A.; Ruotolo, G.; Grp, D.S. Blood lipid levels in relation to glucose status in European men and women without a prior history of diabetes: The decode study. Diabetes Res. Clin. Pract. 2008, $82,364-377$.

33. Sun, X.; Cao, Z.B.; Tanisawa, K.; Ito, T.; Osima, S.; Ishimi, Y.; Tabata, I.; Higuchi, M. Associations between the serum $25(\mathrm{OH}) \mathrm{D}$ concentration and lipid profiles in Japanese men. J. Atheroscler. Thromb. 2014, Available online: http://dx.doi.org/10.5551/jat.26070 (accessed on 27 October 2014).

34. Matthews, D.R.; Hosker, J.P.; Rudenski, A.S.; Naylor, B.A.; Treacher, D.F.; Turner, R.C. Homeostasis model assessment: Insulin resistance and beta-cell function from fasting plasma glucose and insulin concentrations in man. Diabetologia 1985, 28, 412-419.

35. Bonora, E.; Targher, G.; Alberiche, M.; Bonadonna, R.C.; Saggiani, F.; Zenere, M.B.; Monauni, T.; Muggeo, M. Homeostasis model assessment closely mirrors the glucose clamp technique in the assessment of insulin sensitivity: Studies in subjects with various degrees of glucose tolerance and insulin sensitivity. Diabetes Care 2000, 23, 57-63.

(C) 2014 by the authors; licensee MDPI, Basel, Switzerland. This article is an open access article distributed under the terms and conditions of the Creative Commons Attribution license (http://creativecommons.org/licenses/by/4.0/). 